\title{
MELOXICAM-PECTIN- $\beta$-CYCLODEXTRIN TERNARY COMPLEX BY KNEADING FOR ENHANCEMENT OF SOLUBILITY AND DISSOLUTION RATE
}

\author{
RAJESH JAGTAP ${ }^{1 *}$, SHRINIVAS MOHITE ${ }^{2}$ \\ ${ }^{1}$ Department of Pharmaceutics, Annasaheb Dange College of B Pharmacy, Ashta, Sangli, Maharashtra, India. ${ }^{2}$ Department of \\ Pharmaceutical Chemistry, Rajarambapu College of Pharmacy, Kasegaon, Sangli, Maharashtra, India. Email: rajeshjagtap10@gmail.com
}

Received: 29 January 2019, Revised and Accepted: 2 March 2019

\section{ABSTRACT}

Objective: The objective of the present investigation was to improve the solubility and dissolution rate of poorly soluble drug meloxicam by its ternary inclusion complexation with natural polymers and beta-cyclodextrin ( $\beta$-CD) by kneading.

Methods: Equimolar physical mixture (1:1) was prepared by homogeneously kneading drug and $\beta$-CD using a solution of agar and pectin in water to get a paste, then paste was dried overnight to get inclusion complex. Inclusion complex was evaluated for drug content, the yield of the adsorption process, Fourier-transform infrared (FTIR), differential scanning calorimeter (DSC), powder X-ray diffractometry (PXRD), scanning electron microscopy (SEM), dissolution, and stability studies.

Results: The phase solubility diagrams exhibit A showing a linear increase of drug solubility and indicating the formation of soluble complexes. The FTIR and DSC show compatibility between meloxicam and $\beta$-CD, while slight broadening in the peak with a reduction in intensity and early onset indicates the reduction in drug crystallinity which confirms in PXRD pattern. The SEM of binary, as well as ternary, showed no aggregation, and there was a gap between the particles also indicating good redispersibility. The dissolution rate of the drug from the kneaded ternary complex with pectin was significantly rapid compared with the pure drug. The maximum drug release was observed at $85.21 \pm 1.84 \%$ at the end of 60 min. The ternary complex was found stable after 3 months stability studies.

Conclusion: The results indicated that ternary inclusion complexation with natural polymers and $\beta$-CD was most useful for enhancement of solubility and dissolution rate of a poorly soluble drug like meloxicam.

Keywords: Meloxicam, $\beta$-cyclodextrin, Natural polymers, Ternary complex, Solubility enhancement.

(C) 2019 The Authors. Published by Innovare Academic Sciences Pvt Ltd. This is an open access article under the CC BY license (http://creativecommons. org/licenses/by/4. 0/) DOI: http://dx.doi.org/10.22159/ajpcr.2019.v12i4.32126

\section{INTRODUCTION}

Cyclodextrin inclusion complexes play an important role in improving the therapeutic efficacy of drugs with poor solubility and/or stability problems. They are capable of alleviating the undesirable properties of drug molecules through the formation of inclusion complexes [1]. Betacyclodextrin $(\beta-C D)$ is a basic cyclodextrin and hence selected for its higher solubility, and this generally results in more extensive solubilization ability toward lipophilic molecules, with a good safety profile [2]. However, cyclodextrin has problems such as high molecular mass, rather a high cost and potential parenteral toxicity [3]. Strengthening the complexation and solubilization efficacy of cyclodextrins is possible by the use of the ternary agent which may act as a binding agent between cyclodextrin and guest molecule and/or act by solubilizer. Apart from several approaches toward this aim is the addition of suitable auxiliary substances, which can be a useful approach to increase cyclodextrin solubilizing capacity by ternary complex [4-6]. CDs can accommodate a variety of molecules inside the cavity due to its shape to form inclusion compounds. The guest molecules encapsulated by CDs may undergo some changes in their physical, chemical, or biological properties. This feature has been advantageously exploited for increasing the stability and bioavailability of drugs [7-9]. Instead of attempting only binary inclusion complex addition of small amounts of water-soluble polymers to the system, which causes an increase in solubilization efficiency and may result in reducing amounts of $\mathrm{CD}[10,11]$. These results can be attributed to the synergistic effect of polymer and CD solubilization on the formation of drug: CD:water-soluble polymer ternary complexes [12,13]. Water-soluble polymers are able to interact with drugs, CD molecules, and even with the drug: $\mathrm{CD}$ complexes [3]. The mechanism involved in increasing $\mathrm{CD}$ complexation efficiency in the presence of water-soluble polymers is not yet fully understood; however, it is believed that watersoluble polymers can reduce $\mathrm{CD}$ mobility and increase the complex solubility [14]. Cyclodextrin ternary complexes have been tried for overall enhancement of solubility and dissolution rate with carboxylic acid [15,16], amino acids [17,18], sugar alcohol [19], and hydrophilic polymer $[20,21]$. There are different types of the hydrophilic polymer have been tried including synthetic and semi-synthetic, but there was a rare use of natural polymer for this purpose [22].

Meloxicam (4-hydroxy-2-methyl- $N$-\{5-methyl-2-thiazolyl\}- $H-1, \quad 2$ benzothiazine-3-carboxamide 1, 1-dioxide) is a potent nonsteroidal anti-inflammatory drug. It is practically insoluble in water $\left(12 \mathrm{mg} / \mathrm{mL}^{1}\right)$. Its poor solubility and wettability lead to difficulties in oral and parenteral formulations. In the present investigation, attempt was made to improve solubility and dissolution rate of the poorly soluble drug using ternary complexation with $\beta-\mathrm{CD}$ and natural polymers such as agar and pectin as natural polymers have several advantages over synthetic or semi-synthetic polymers.

\section{MATERIALS AND METHODS}

Material

Meloxicam was obtained as a gift sample from Cipla Ltd., Mumbai, India. $\beta$-CD was gifted by Lupin Ltd Pune. All other chemicals and solvents used were of pharmaceutical and analytical grade. Double distilled water was used throughout the study for all the experimental procedures.

Phase solubility studies

The solubility behavior of meloxicam was examined in distilled water at room temperature $\left(25 \pm 2^{\circ} \mathrm{C}\right)$ according to the method described by Higuchi 


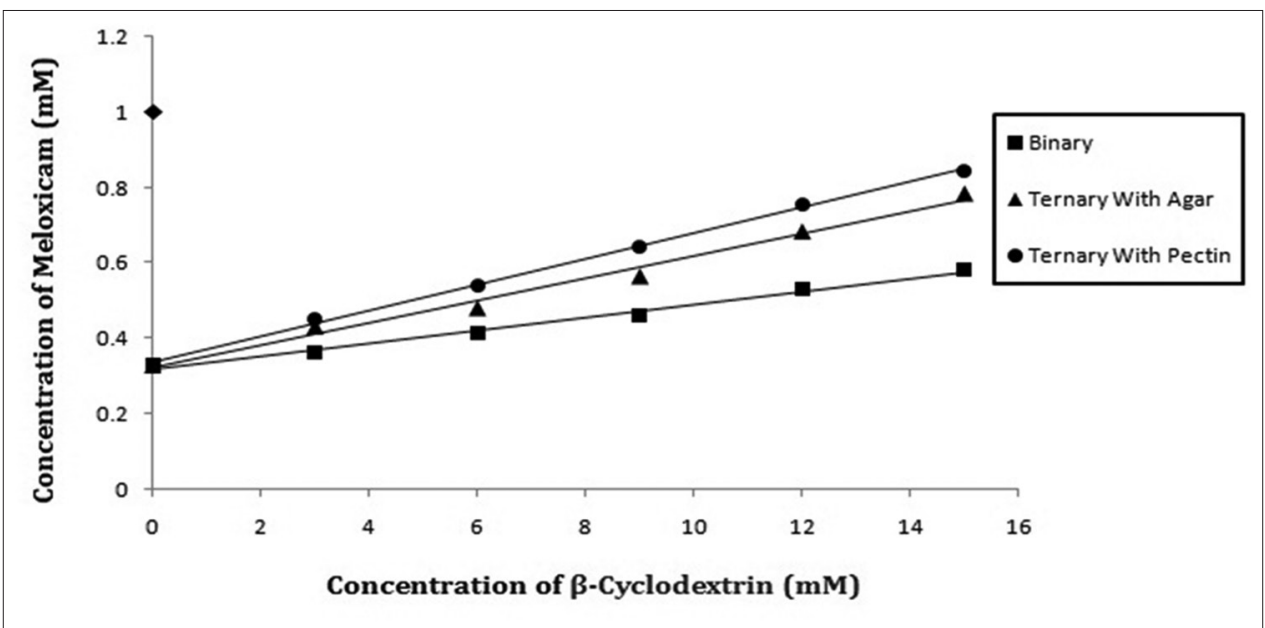

Fig. 1: Phase solubility diagram of meloxicam beta-cyclodextrin ( $\beta$-CD) binary system, meloxicam $\beta$-CD -Agar ternary system, and meloxicam $\beta$-CD- Pectin ternary system

and Connors 1965 [23]. Known excess amounts of ME (50 mg) were added to $20 \mathrm{~mL}$ of aqueous solutions of various concentrations of $\beta$-CD ranging between $1 \mathrm{mM}$ and $15 \mathrm{mM}$. The suspensions were vigorously shaken in a shaking water bath at $25 \pm 0.5^{\circ} \mathrm{C}$ for 2 days. After equilibrium was attained, saturated solutions were centrifuged, and the supernatants were filtered through a 50-mm Millipore filter. After suitable dilution, samples were assayed for ME concentration using a uitraviolet (UV) spectrophotometer (Shimadzu UV spectrophotometer 1800) at $\lambda_{\max }$ of $360 \mathrm{~nm}$ against blank prepared in the same concentration of $\beta-\mathrm{CD}$. To establish the effect of natural polymers (pectin and agar) on the solubility diagram, each was added separately at a concentration of $0.5 \%(\mathrm{~m} / \mathrm{V})$ to a suspension of ME- $\beta C D$ and then allowed to equilibrate at $25 \pm 0.5^{\circ} \mathrm{C}$ for 2 days. The each solubility was investigated using the previously described method and performed in triplicate. The phase solubility diagram was constructed by plotting the dissolved Meloxicam concentration against the respective concentration of $\beta$-CD. The binding constant Ka was calculated from a phase solubility diagram using its slope and intercept values [24]. Phase solubility study graph is given in Fig. 1.

\section{PREPARATION OF SOLID COMPLEXES}

\section{Preparation of binary complex by kneading}

In this method, the equimolar physical mixture of $1: 1$ was prepared by homogeneously blending exactly weighed amounts of drug and $\beta$-CD until the homogenous mixture is obtained and 1.5 times of water to the total weight of the physical mixture was added slowly during continuous kneading. The mixture is kneaded for about $1 \mathrm{~h}$ to get the paste. Then, this paste was allowed to dry at room temperature for $24 \mathrm{~h}$ in dark place to protect it from light, and then the dried powder sieved to get uniform particle size distribution [25].

\section{PREPARATION OF TERNARY COMPLEX BY KNEADING}

Ternary inclusion complexes were prepared by slight modification in the above mention method to add a ternary agent that is a natural hydrophilic polymer. An equimolar physical mixture of $1: 1$ was prepared by homogeneously blending exactly weighed amounts of drug and $\beta$-CD until the homogenous mixture is obtained and sufficient volume of $0.5 \%$ aqueous solution of natural polymer Pectin and Agar was added slowly during continuous kneading to get ternary complexes. The mixture is kneaded for about $1 \mathrm{~h}$ to get the paste. Then, this paste was allowed to dry at room temperature for $24 \mathrm{~h}$ in dark place to protect it from light, and then the dried powder sieved to get uniform particle size distribution [25].

\section{Characterization of solid complexes}

\section{Drug content and yield of kneading process}

Kneaded solid complex ( $10 \mathrm{mg}$ ) was weighed accurately and extracted using $100 \mathrm{~mL}$ of phosphate buffer $\mathrm{pH} 6.8$ by shaking for $12 \mathrm{~h}$ on a

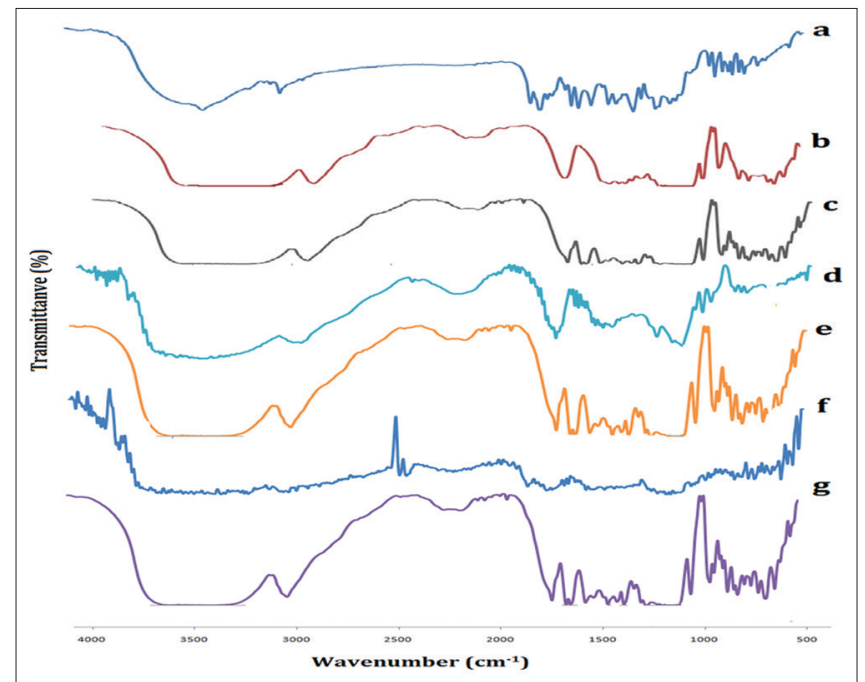

Fig. 2: Fourier transform infrared spectra of (a) pure meloxicam, (b) pure $\beta$-cyclodextrin and (c) meloxicam beta-cyclodextrin $(\beta-C D)$ binary complex (d) pure agar (e) meloxicam $\beta$-CD-agar ternary complex (f) Pure pectin (g) meloxicam $\beta$-CD-pectin ternary complex

rotary shaker. After shaking the sample filter through Whatman filter paper and after sufficient dilutions, samples were analyzed spectrophotometrically at $238 \mathrm{~nm}$ (Shimadzu UV spectrophotometer 1800). Drug content was calculated from the standard curve of meloxicam in phosphate buffer $\mathrm{pH} 6.8$ [26] yield of the kneading process was determined by the corelating weight of added solute to that of recovered one.

Fourier transform-infrared (FT-IR) spectrophotometer studies FT-IR has been employed as a useful tool to identify drug excipient interaction. Samples were analyzed by the potassium bromide pellet method in an IR spectrophotometer (Alpha T Bruker) in the region from 4000 to $400 \mathrm{~cm}^{-1}$. Pure meloxicam, binary solid complexes and ternary solid complexes with agar and pectin were evaluated by comparing its FT-IR spectra. FT-IR spectra of Pure nifedipine and adsorbed product are shown in Fig. 2.

Differential scanning calorimeter (DSC) analysis

DSC has been one of the most widely used calorimetric techniques to study the solid state interaction of the drug with other ingredients [27]. Samples of the pure drug and adsorbed product were taken in flat- 
bottomed aluminum pans and heated over a temperature range of 30$300^{\circ} \mathrm{C}$ at a constant rate of $10 / \mathrm{min}$ with purging of nitrogen $(50 \mathrm{ml} / \mathrm{min})$ using alumina as a reference standard in a DSC (Mettler Toledo, Staresw 920). The DSC thermogram of pure meloxicam and kneaded solid complexes is shown in Fig. 3.

Powder X-ray diffractometry (PXRD) analysis

The powder X-ray diffraction technique has been extensively utilized to study the interaction and to obtain the changes in the crystallinity of the kneaded product. PXRD study was carried out using X-ray diffractometer (Miniflex 600 X-ray diffractometer, Rigaku corporation Japan). For this the samples of pure drug and kneaded product were irradiated with monochromatized $\mathrm{CuK} \alpha$ radiation and analyzed from 5 to $60(2 \theta)$ [28]. The PXRD diffractograms of pure meloxicam and kneaded solid complexes are shown in Fig. 4.

\section{Scanning electron microscopy (SEM) studies}

The surface morphology of pure meloxicam and kneaded solid complexes was observed using a SEM (VEG A3 TESCAN), under accelerating voltage of $15 \mathrm{keV}$. Samples were fixed on SEM stub with double-sided adhesive tape and then coated in a vacuum with a thin gold layer before investigation [29]. The SEM images of pure meloxicam and kneaded solid complexes are shown in Fig. 5.

\section{DISSOLUTION STUDIES IN PHOSPHATE BUFFER}

An accurately weighed amount of pure meloxicam, binary solid complex, and ternary solid complexes with agar and pectin equivalent to $15 \mathrm{mg}$ of added to the dissolution medium. The dissolution study was performed in phosphate buffer $\mathrm{pH}$ 7.4. The dissolution study was carried out using USP apparatus II (EDT 08LX Electrolab) at $37 \pm 0.5^{\circ} \mathrm{C}$ and $100 \mathrm{rpm}$ paddle speed. The samples were withdrawn from dissolution media at specified time interval up to $120 \mathrm{~min}$, and the absorbance of the sample was recorded using UV (Shimadzu UV spectrophotometer 1800) at $\lambda_{\max }$ of $360 \mathrm{~nm}$ against blank [30]. Dissolution profiles are shown in Fig. 6.

\section{Stability study}

Stability study for meloxicam ternary complex with pectin by kneading was carried out with the help of stability chamber (Remi SC-19 Plus) by storing $1 \mathrm{gm}$ of the above sample in an ambered colored screw-capped glass bottles at accelerated and controlled temperatures $40^{\circ} \mathrm{C}$ and relative humidities (75\%) for 3 months [31-33]. The physical mixture was evaluated for physical appearance and in vitro dissolution at the end of 3 months.

\section{RESULTS AND DISCUSSION}

\section{Phase solubility studies}

Phase solubility diagrams of ME in the binary system with $\beta-C D$, a ternary system with $\beta$-CD agar and $\beta$-CD pectin in aqueous solutions at $25^{\circ} \mathrm{C}$ is illustrated in Fig. 1. Solubility diagrams exhibit $\mathrm{A}_{\mathrm{t}}$ type according to the Higuchi and Connors classification [23], showing a linear increase of drug solubility on increasing $\beta$-CD concentration and indicating the formation of soluble complexes. The slopes of the curve were found to be $0.00644,0.0119$, and 0.0129 in case of binary complex, ternary complex with agar, and ternary complex with pectin, respectively. A slope value of $<1.00$ indicates the formation of an inclusion complex in a molar stoichiometric ratio of $1: 1$ between the meloxicam and $\beta$-CD in a solution of the hydrophilic natural polymeric solution. The ratio between the slopes of the phase solubility curves of ternary and binary systems, as an index of the relative solubilizing efficiency, was 1.87 for agar and 2.02 for pectin, confirming the greater effectiveness of the ternary system. The stability constant (Ks) computed from the slope and intercept of the phase solubility diagram was found to be $587.24 \mathrm{M}^{-1}, 912.23 \mathrm{M}^{-1}$, and $1021.58 \mathrm{M}^{-1}$ in case of the binary system, ternary system with agar, and ternary system with pectin indicating an increase in stability constant value of ternary system compared to the binary system. The mechanism involved in enhancing the solubility of meloxicam may be due to the synergistic effect of solubilization effect of natural hydrophilic polymer and inclusion complexation of ME with $\beta$-CD.

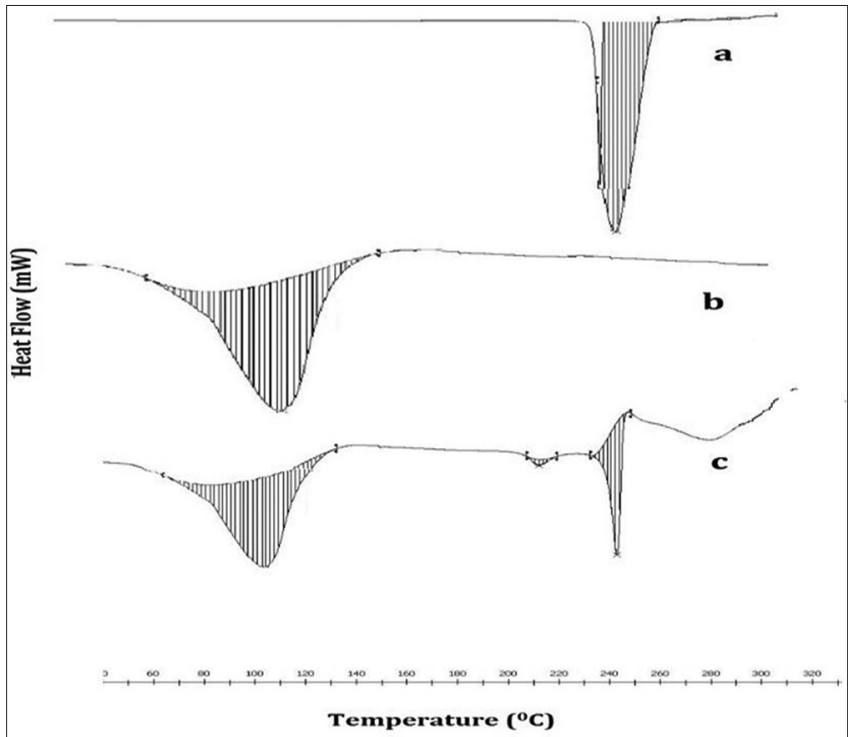

Fig. 3: DSC thermograms of (a) pure meloxicam, (b) pure betacyclodextrin $(\beta-C D)$, and $(c)$ meloxicam- $\beta-C D$-pectin ternary complex

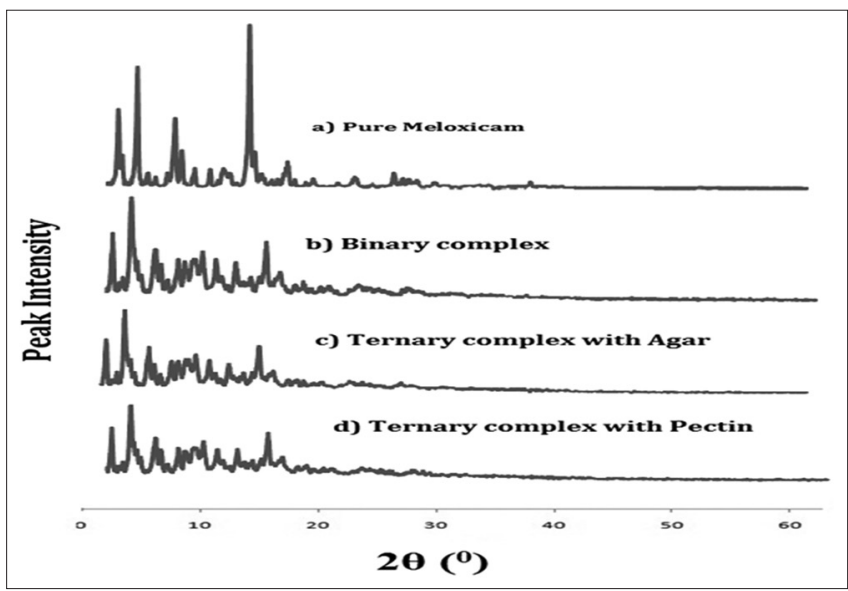

Fig. 4: Powder X-ray diffractometry diffractograms for (a) pure meloxicam, (b) binary complex (c) ternary complex with agar

(d) ternary complex with pectin
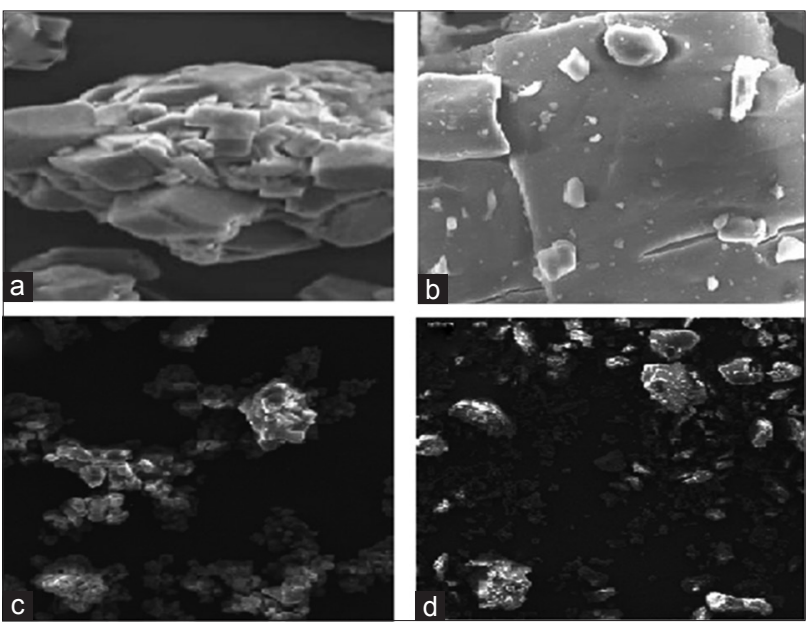

Fig. 5: SEM images of (a) pure meloxicam (b) pure betacyclodextrin (c) binary complex, and (d) ternary complex with pectin 
Drug content and yield of kneading process

The drug content of binary solid complex and ternary solid complexes was determined using a previously reported method [26]. The practical drug content of binary solid complex, ternary complex with agar, and ternary complex with pectin was found to be $22.10 \pm 0.23,21.85 \pm 0.47$, and $21.90 \pm 0.42$, respectively, which corresponded to the ratio of the drug with $\beta$-CD and a natural hydrophilic polymer. The yield of the kneading process was found more than $98 \%$ which indicates negligible loss during kneading and its recovery after overnight drying. The greater yield of the kneading process as compared to another method of complexation like spray drying makes a suitable method for preparation of binary and ternary complexation. There was no any significant difference in complexation efficiency between kneading method and spray drying method reported by the previous researcher [15] confirms kneading method is a suitable method for formation of inclusion complex as it is time saving and simple. The theoretical drug content, practical drug content, and yield of the kneading process are summarized in Table 1.

\section{FT-IR spectrophotometer studies}

The FT-IT analysis has been carried out to study the interaction between meloxicam with $\beta$-CD and natural hydrophilic polymers and to study complexation by kneading. The pure meloxicam showed characteristic at $1620 \mathrm{~cm}^{-1}$ (C=0 Stretching for Amide), $3292 \mathrm{~cm}^{-1}$ (N-H Stretching for Amide, $2923 \mathrm{~cm}^{-1}$ (Ar- CH Stretching), $1185 \mathrm{~cm}^{-1}$ and $1346 \mathrm{~cm}^{-1}$ for $\mathrm{S}=0$ Stretching vibration of sulfonamide group, $1267 \mathrm{~cm}^{-1}$ for C-N Stretching vibration of Aliphatic amine, etc., confirming the earlier reported peaks. The FTIR spectra of $\beta$-CD were characterized by an intense band between $3700 \mathrm{~cm}^{-1}$ and $3000 \mathrm{~cm}^{-1}$ (OH Stretching), a peak at 2917.77 $\mathrm{cm}^{-1}$ (Aliphatic CH Stretching), a broad peak at $1635.34 \mathrm{~cm}^{-1}$, and a band with distinct peaks in the region between 1200 and $1000 \mathrm{~cm}^{-1}$. Typical principle peaks not showed any shift in IR spectra in binary or ternary solid complex, confirmed that there is no interaction between $\beta-C D$, natural hydrophilic polymer, and guest molecule. The bending vibrations of the aromatic ring were not observed in the spectra of the kneaded product either in binary or ternary solid complex, indicating that the vibrating and bending of the guest molecule were restricted due to formation of inclusion complex, this dramatic change was probably related to the formation of intermolecular hydrogen bonds between the guest and host molecules [34]. The FTIR spectra of meloxicam- $\beta$-CD kneading binary solid complex and its ternary complexes with agar and pectin not showed any new peak, indicating no chemical bond were created in the formed compound. The FI-IR spectra of pure meloxicam, binary solid inclusion complex, and ternary solid inclusion complex with the natural hydrophilic polymer are shown in Fig. 2.

\section{DSC analysis}

DSC analyses were performed to evaluate the thermal behavior of meloxicam, in the presence of $\beta-C D$ and natural polymers used in binary and ternary solid inclusion complexes. When guest molecule incorporated into cyclodextrin central cavity, their melting point shifts to the different temperature, or it may disappear. One sharp endothermic peak was observed near around $254^{\circ} \mathrm{C}$ with onset at $248^{\circ} \mathrm{C}$ contributed to the melting point of crystalline meloxicam in the thermogram of pure drug. Meloxicam- $\beta$-CD-pectin ternary complex showed onset $247^{\circ} \mathrm{C}$ and peak at $252^{\circ} \mathrm{C}$ with slight broadening in peak as well reduction in intensity and early onset as compared to the pure drug indicates a reduction in drug crystallinity and conversion in the amorphous form which is the positive effect for solubility enhancement. The shift in the dehydration band of $\beta$-CD in the spectra of the kneaded product can be attributed to the formation of inclusion complex of the drug with $\beta$-CD. The reduction in the enthalpy of fusion of the drug has been reported to be an indication for the formation of an inclusion complex with $\beta$-CD. The DSC thermograms of pure meloxicam, pure $\beta-C D$, and ternary solid complex are shown in Fig. 3.

\section{PXRD analysis}

The powder X-ray diffraction spectroscopy was used to asses the degree of crystallinity of the given sample due to complexation of the poorly soluble drug by kneading. When the drug goes to the cavity of $\beta$-CD and forms soluble inclusion complex, overall crystallinity of drug decreases and with an increases in amorphous nature. Pure meloxicam showed numerous distinctive peaks at 14.96, 25.86, 25.90, etc., with high peak intensity that indicated a high crystallinity; however, peak intensity decreased in the binary complex of drug with $\beta$-CD indicating conversion of the drug in amorphous form. The peak intensity further decreased in a ternary complex with agar as compared to the binary complex and observed very less in case of the ternary complex with pectin indicates the addition of ternary agent is beneficial to decrease crystallinity of a drug. The conversion of crystalline nature of drug to amorphous nature of help in increasing its solubility in different $\mathrm{pH}$

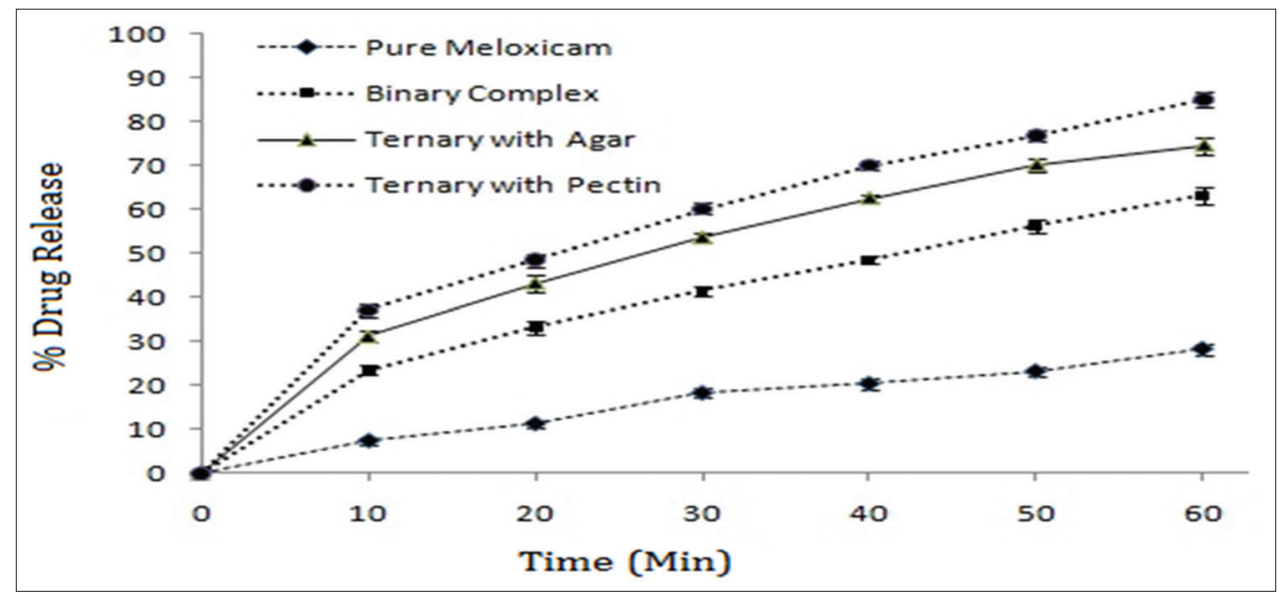

Fig. 6: Dissolution studies of pure meloxicam, binary complex, ternary complex with agar, and ternary complex with pectin

Table 1: Drug content and yield of kneading process for the binary and ternary complex

\begin{tabular}{lll}
\hline Solid complexes (1:1 molar stoichiometric ratio) & $\begin{array}{l}\text { Theoretical drug } \\
\text { content (\%) }\end{array}$ & $\begin{array}{l}\text { *Practical drug } \\
\text { content (\%) }\end{array}$ \\
\hline Binary complex & 23.64 & $22.10 \pm 0.23$ \\
Ternary with agar & 23.41 & $21.85 \pm 0.47$ \\
Ternary with pectin & 23.41 & $21.90 \pm 0.42$ \\
\hline
\end{tabular}

*Represents mean \pm Standard deviation $(\mathrm{n}=3)$ 
conditions of GI tract. Significant reduction in peak intensities in PXRD pattern was observed in case ternary complex in the presence of natural hydrophilic polymer, as compared to the pure drug was attributed to the synergistic effect of $\beta$-CD and agar or pectin. PXRD diffractograms of pure meloxicam, binary complex, and ternary complex with agarand ternary complex with pectin are shown in Fig. 4.

\section{SEM studies}

The SEM study was done to check surface morphology of the meloxicam particles and its relevant changes when complexed as a binary and ternary inclusion complex using the kneading method. Pure meloxicam image shows crystalline rectangular shape particles, whereas an image of the solid binary complex of the drug does not show any crystalline material indicating kneading of meloxicam with $\beta$-Cd in binary complex helps to convert crystalline form of the drug to amorphous form. The similar types of results observed in SEM images of ternary kneading complex that is drug converted to the amorphous form which was a positive effect for the enhancement of solubility. Pure meloxicam shows crystalline rectangular shape while $\beta$-CD was observed to be irregular in shape, but this original morphology of both components had disappeared in binary and ternary solid inclusion complex. These changes suggested the formation of inclusion complex; moreover, SEM of binary as well as ternary showed no aggregation, and there was a gap between the particles also, indicating good redispersibility. The SEM images of pure meloxicam, pure $\beta$-Cd, binary inclusion complex, and ternary inclusion complex with pectin are shown in Fig. 5.

\section{In vitro dissolution studies in phosphate buffer}

The dissolution study was carried out in phosphate buffer $\mathrm{pH} 7.4$ for comparison of percent drug release of pure meloxicam with binary and different ternary inclusion complex. Pure meloxicam showed drug dissolution of $18.87 \pm 0.90 \%$ in 30 min while meloxicam- $\beta$-Cd binary complex showed the dissolution of $41.56 \pm 1.52 \%$ in $30 \mathrm{~min}$. The percent drug release dissolution further increased in the ternary complex with agar as well as pectin and which was found to be $53.64 \pm 1.88$ and $60.21 \pm 1.54$, respectively. The maximum drug release was found to be $85.21 \pm 1.84 \%$ at the end of $60 \mathrm{~min}$ from meloxicam ternary complex with pectin which was highest drug release compared to ternary complex with agar as well as binary complex indicating ternary complex with natural hydrophilic polymer pectin showed better drug dissolution in phosphate buffer 6.8. Increase in the dissolution rate was recorded for solid complexes compared to the pure drug. It can be assumed that, in the dissolution process, $\beta$-Cd molecules will operate locally surrounding the drug particles. This action resulted in the rapid increase in the amount of dissolved drug. The order of drug dissolution was found to be ternary complex with pectin > ternary complex with agar $>$ binary complex $>$ pure meloxicam indicating that the ternary complex with pectin is more promising for the enhancement of solubility as well as drug dissolution of meloxicam. The increase in drug dissolution is the result of the synergistic action of $\beta-C D$ and the natural hydrophilic polymer. The dissolution profiles of pure meloxicam, binary complex, ternary complex with agar, and pectin in phosphate buffer 7.4 are shown in Fig. 6.

\section{Stability studies}

There was no significant change in the physical appearance and percent drug dissolution in the kneaded product of meloxicam. A stability results indicate that kneaded product was sufficiently stable under accelerated and controlled conditions. No change in physical appearance and other parameters indicates that solid complex product was found stable under accelerated temperature condition as it gives more protection to the drug as compared to drug alone as drug enclosed in the cavity of $\beta$-CD.

\section{CONCLUSION}

The present study determined the utility ternary complexation with the natural hydrophilic polymer to enhance the solubility and dissolution rate of the BCS Class-II drug meloxicam. Dissolution study indicates that ternary complex with pectin is most useful for enhancement of solubility of meloxicam as compare to the drug alone. Meloxicam- $\beta$ Cdpectin ternary kneaded product can be used for further development of an oral solid drug delivery system to overcome the solubility problem.

\section{ACKNOWLEDGMENT}

Authors are thankful to the Cipla Ltd., Mumbai for providing gift sample of meloxicam. Shivaji University, Kolhapur and D Y Patil University Kolhapur are acknowledged for assistance with analytical work.

\section{AUTHOR'S CONTRIBUTIONS}

The author finds out research gap for proposed work, finalized the methodology, collected the data, performed, and interprets the results of analysis work.

\section{CONFLICTS OF INTEREST}

The authors declare that they have no conflicts of interest.

\section{REFERENCES}

1. Shah N, Seth AK, Balaraman R. Bioavailability enhancement of poorly soluble raloxifene by designing inclusion complex with B-cyclodextrin. Int J Pharm Pharm Sci 2015;7:205-11.

2. Prabhakaran R, Janakiraman K, Harindran J. Improvement of bioavailability of cefuroxime axetil oral suspension by inclusion complexation method. Int J Pharm Pharm Sci 2016;8:361-4.

3. Loftsson T, Brewster ME. Pharmaceutical applications of cyclodextrins 1. Drug solubilization and stabilization. J Pharm Sci 1996;85:1017-25.

4. Baboota S, Agarwal SP. Inclusion complexation of meloxicam with b-cyclodextrin. Indian J Pharm Sci 2002;64:408-11.

5. Loftsson T. Increasing the cyclodextrin complexation of drugs and drug biovailability through addition of water-soluble polymers. Pharmazie 1998;53:733-40.

6. Vijaya Kumar SG, Mishra DN. Preparation, characterization and in vitro dissolution studies of solid dispersion of meloxicam with PEG 6000. Yakugaku Zasshi 2006;126:657-64.

7. Uekama K, Hirayama F, Irie T. Application of cyclodextrins. Drug Target Deliv 1994;3:411-56.

8. Jagtap S, Magnum C, Jadge D, Jagtap R. Solubility enhancement technique: A review. J Pharm Sci Res 2018;10:2205-11.

9. Loftsson T, Brewster M. Cyclodextrins as pharmaceutical excipient. Pharm Tech Eur 1997;9:26-34.

10. Arora P, Singh J, Chadha R. Physicochemical characterization and evaluation of telmisartan: Hydroxypropyl-B-cyclodextrin: Tween 80 inclusion complex. Int J Pharm Pharm Sci 2017;9:51-8.

11. Mura P, Faucci MT, Bettinetti GP. The influence of polyvinylpyrrolidone on naproxen complexation with hydroxypropyl-beta-cyclodextrin. Eur J Pharm Sci 2001;13:187-94.

12. Shaikh AA, Chaudhari PD, Holkar SS. A design of experiment approach for optimization and characterization of Etodolac ternary system using spray drying. Int J Pharm Pharm Sci 2017;9:233-40.

13. Brewster M, Loftsson T. Cyclodextrins as pharmaceutical solubilizer. Adv Drug Deliv Rev 2007;59:645-66.

14. Valero M, Carrillo C, Rodríguez LJ. Ternary naproxen: $\beta$-cyclodextrin: Polyethylene glycol complex formation. Int J Pharm 2003;265:141-9.

15. Pokharkar V, Khanna A, Venkatpurwar V, Dhar S, Mandpe L. Ternary complexation of carvedilol, beta-cyclodextrin and citric acid for mouthdissolving tablet formulation. Acta Pharm 2009;59:121-32.

16. Rakkaew P, Suksiriworapong J, Chantasart D. B-cyclodextrin-based ternary complexes of haloperidol and organic acids: The effect of organic acids on the drug solubility enhancement. Pharm Dev Technol 2018; 23:715-22

17. Mura P, Maestrelli F, Cirri M. Ternary systems of naproxen with hydroxypropyl-beta-cyclodextrin and aminoacids. Int J Pharm 2003; 260:293-302.

18. JagtapS,MagdumC.EnhanceddissolutionandsolubilityofEpalrestatwith $\beta$-cyclodextrin ternary complex using Arginine. J Drug Deliv Ther 2018; 8:62-7.

19. Ghorpade VS, Remeth D, Kailas M, Vijay H. Preparation and evaluation of domperidone/ $\beta$-cyclodextrin/citric acid/mannitol quaternary inclusion complex: An in vitro study. Asian J Pharm 2016;10:375-85.

20. El-Maradny HA, Mortada SA, Kamel OA, Hikal AH. Characterization of ternary complexes of meloxicam-HPbetaCD and PVP or L-arginine 
prepared by the spray-drying technique. Acta Pharm 2008;58:455-66.

21. Jug M, Bećirević-Laćan M. Multicomponent complexes of piroxicam with cyclodextrins and hydroxypropyl methylcellulose. Drug Dev Ind Pharm 2004;30:1051-60.

22. de Miranda JC, Azevedo Martins TE. Cyclodextrins and ternary complexes: Technology to improve solubility of poorly soluble drugs. Braz J Pharm Sci 2011;47:665-82.

23. Higuchi T, Connors KA. Phase-solubility techniques. Adv Anal Chem Instrum 1965;4:117-212.

24. Bayomi MA, Abanumay KA, Al-Angary AA. Effect of inclusion complexation with cyclodextrins on photostability of nifedipine in solid state. Int J Pharm 2002;243:107-17.

25. Bhosale Ashok V, Hardikar Sharwaree R, Jagtap Rajesh S. Formulation of beta-cyclodextrin complexed controlled release matrix tablet of glipizide and its in vitro evaluation. Int J Pharm Tech Res 2009;1:773-8.

26. Sharma S, Sher P, Badve S, Pawar AP. Adsorption of meloxicam on porous calcium silicate: Characterization and tablet formulation. AAPS PharmSciTech 2005;6:E618-25.

27. Ramana MV, Himaja M, Dua K. A new approach: Enhancement of solubility of rofecoxib. Asian J Pharm 2008;2:96-101.

28. Streubel A, Siepmann J, Bodmeier R. Floating matrix tablets based on low density foam powder: Effects of formulation and processing parameters on drug release. Eur J Pharm Sci 2003;18:37-45.

29. Rajesh J, Rajendra D, Shrinivas M. Enhancement of solubility and dissolution rate of nifedipine by using novel solubilizer sepitrap 80 and sepitrap 4000. J Drug Deliv Ther 2018;8:293-300.

30. Tran HT, Park JB, Hong KH, Choi HG, Han HK, Lee J, et al. Preparation and characterization of $\mathrm{pH}$-independent sustained release tablet containing solid dispersion granules of a poorly water-soluble drug. Int J Pharm 2011;415:83-8.

31. International Conference on Harmonization (ICH). Harmonized Tripartite Guideline for Stability Testing of New Drugs Substances and Products Q1A (R2) Aug-2003. Q1 (R2). International Conference on Harmonization; March, 2004.

32. Rhodes CT, Cartesan T. Drug Stability Principle and Procedure. $3^{\text {rd }}$ ed. New York: Marcel Dekker; 2001.

33. Yi T, Wan J, Xu H, Yang X. A new solid self-microemulsifying formulation prepared by spray-drying to improve the oral bioavailability of poorly water soluble drugs. Eur J Pharm Biopharm 2008;70:439-44.

34. Gohel MC, Patel MR, Patel KV. Studies in dissolution enhancement of nifedipine. Drug Dev Ind Pharm 1996;22:263-8. 